\title{
La gestión de la comunicación
}

\section{institucional interna}

en las universidades de

la Comunidad de Madrid

Fortalezas y debilidades

The Management of

Internal Institutional

Communication at

the Universities of the

Community of Madrid

Strengths and Weaknesses

Dr. Arturo Gómez Quijano

Profesor Asociado de la Universidad Complutense de Madrid

artgomez@ucm.es

Dra. Lelia Zapata Palacios

Profesora Universidad Complutense de Madrid leliazap@ucm.es
Gómez Quijano, A. y Zapata Palacios, L. (2017)

La gestión de la comunicación institucional interna en las universidades de la Comunidad de Madrid: fortalezas y debilidades

Revista Internacional de Investigación en Comunicación aDResearch ESIC. № 16 Vol 16

Segundo semestre, julio-diciembre 2017 · Págs. 60 a 77 DOI: 16.7263/ADRESIC.016.003 
RESUMEN

\section{Clasificación JEL:}

123, M12, M14

Palabras clave:

Comunicación

corporativa,

comunicación

interna,

universidad,

calidad de la educación
Este artículo se centra en el perfil del comunicador interno en las universidades de la Comunidad de Madrid. Realiza un estudio descriptivo que incluye dos tipos de ítems: información sobre lo que estos profesionales son e información sobre las metodologías, recursos, herramientas y funciones que afirman desempeñar. Para sus reflexiones toma como referencia un estudio de investigación cuantitativo aplicado a 14 responsables de comunicación interna de universidades del sector público y privado, que operan en la Comunidad de Madrid. La investigación, que utiliza una metodología descriptiva, concluye que la tarea de gestionar la comunicación interna en las instituciones universitarias es una responsabilidad asumida por parte de las universidades madrileñas y que su gestión va en ascenso respecto a la situación existente en 2005; la función se encuentra en fase de avance, presenta fortalezas aunque también, un número considerable de debilidades, siendo la mayor, la limitación los recursos presupuestarios. El perfil que lidera esta área es un titulado universitario mayoritariamente periodista, publicista o relaciones públicas. Sin embargo, nos encontramos ante un profesional multitarea ya que, además de gestor, se desempeña como docente. Por otro lado, es necesario que desde el área de comunicación interna se ponga el foco en objetivos y proyectos que mejoren el sentido de pertenencia, la calidad educativa y una cultura digital basada en la colaboración.

\section{ABSTRACT}

\section{JEL Classification: \\ 123, M12, M14 \\ Key words:}

Corporate communication, internal public relations, university, educational quality
This article focuses on the profile of the internal communicator at the universities of the Community of Madrid. It performs a descriptive study that includes two items: information about what these professionals are and information about the methodologies, resources, tools and functions they claim to carry out. For these reflections, a quantitative research study applied to 14 internal communication officers of public and private universities operating in the Community of Madrid are taken as reference. The research, which uses a descriptive methodology, concludes that the task of managing the internal communication at university institutions is a responsibility assumed by the Madrid universities and that its management is rising compared to the situation in 2005; The function is in stage of development, there are strengths, but also a considerable number of weaknesses, the greater being the limitation in budgetary resources. The profile that leads this area is a university degree mostly related to journalism, publicity or public relations. However, we are faced with a multi-tasking professional since, apart from being a manager, they are also a professor. On the other hand, it is necessary for the internal communication area to focus on objectives and projects that improve the sense of belonging, educational quality and a digital culture based on collaboration. 


\section{Introducción}

La comunicación interna (en adelante CI) sigue ganando protagonismo en la mayor parte de empresas e instituciones españolas, según lo demuestran los estudios realizados por el observatorio responsable (Observatorio de comunicación interna, 2009, 2012). A juzgar por el número de organizaciones que integran en sus estructuras áreas y/o departamentos de comunicación interna, podemos decir que esta disciplina sigue ganando terreno, en empresas e instituciones (Dircom, 2010).

La comunicación interna progresa porque ya se le valora como una gestión estratégica imprescindible para que la entidad alcance sus objetivos. Sin embargo, es preciso revisar y analizar qué objetivos son prioritarios para cada organización y asignar los recursos económicos necesarios para que pueda alcanzarlos (Saló, 2005).

En la práctica, ocurre muchas veces que sus responsables centran sus objetivos en desempeñar labores informativas, dejando relegado el objetivo de mejorar el sentido de pertenencia, haciendo énfasis en la identidad. El discurso de identidad corporativa debe según Carrascosa, incluir cuatro facetas: 1. La personalidad: quienes somos, 2. La misión: qué hacemos 3. La visión: por qué lo hacemos, a dónde queremos llegar y 4. El compromiso: qué puedo hacer yo para alcanzar la visión (Carrascosa, 2003).

Las personas deben estar en el centro de cualquier estrategia de comunicación interna; al público interno hay que empoderarlo (García Giménez, 1999). Sin embargo, este objetivo se omite en la universidad madrileña, hecho que puede afectar a la calidad educativa y al prestigio de la universidad; los públicos internos de una universidad están llamados a convertirse en embajadores de la marca universidad, y en este sentido, la comunicación interna puede avivar el contrato psicológico que vincula a los hombres con las organizaciones.

Comunicar significa en las organizaciones compartir ideas, intercambiar sentimientos, opiniones y pensamientos, valores institucionales (Gondrand, 1978). En la universidad y en cualquier organización, la comunicación interna cumple un rol de facilitador como elemento constructor y difusor de una misión, visión y valores que todo el personal debería conocer, hacer suyo y sentir orgullo de pertenencia. De esta manera, ayuda además, a las instituciones a promover un proyecto de excelencia orientado a la calidad.

Como bien advierte Palencia-Lefler Ors, Manuel en su artículo: «La incomunicación interna en la universidad española» esta institución tiene muchas contradicciones entre la Dirección, por falta de voluntad, o negligencia.

Esta omisión es delicada ya que si la universidad descuida la comunicación o simplemente, no fluye, la gestión de la calidad educativa está amenazada, se rompe el ligadura entre sus actores: personal administrativo, alumnos, profesores y autoridades universitarias (Palencia- Lefler, 2008).

En la era de la sociedad conectada, la universidad madrileña debería estar en proceso de metamorfosis, mejorando su calidad educativa ya que necesita competir en el mercado global siendo consciente del perfeccionamiento paulatino alcanzado por los contenidos e-learning.

En los próximos años las palancas de desarrollo del e-commerce serán las web específicas para móviles: msites, el aumento de apps, donde la formación e-learning se verá favorecida por la demanda de sus servicios (Zapata, 2016:32). 


\section{Antecedentes y estado de la cuestión}

Hemos constatado que existe un antecedente de estudio de investigación referido a la gestión de la comunicación interna en las universidades madrileñas, desde la perspectiva del marketing; se titula: Análisis del Marketing interno en las universidades de la Comunidad de Madrid (Romero y Calero, 2005). La investigación realizada y disponible, ofrece limitados detalles de su estudio, tampoco establece unas conclusiones diferenciadas de la comunicación interna entre universidades públicas y privadas; contiene información genérica desde la perspectiva del marketing, planteamiento comercial que no es objetivo de nuestra investigación. Para concluir, el marketing interno y la comunicación interna van tomando cuerpo, a pesar de sus dificultades en su implantación (Romero y Calero 2005).

Por nuestra parte, entendemos que la comunicación interna no forma parte del marketing, sino que, como sentencia el profesor Martín:

La comunicación interna debe estar alineada con la estrategia de la organización y al plan de comunicación externa; necesita estar siempre interconectadas e incorporadas en el plan o estrategia de comunicación de toda organización, ya sea una empresa o una institución (Martín Martín, 2012:58).

Sobre la comunicación interna en las instituciones universitarias en regiones españolas, hemos constatado que existen otros dos estudios regionales en la Comunidad Valenciana y Andalucía, en estos casos se abordan desde tesis doctorales, poniendo énfasis en la comunicación digital.

- 2016 Comunidad Andaluza. Esta tesis doctoral de José Simón Onieva titulada Gabinetes de comunicación universitarios y redes so- ciales: estudio del uso de las redes sociales por las universidades públicas andaluza, concluye que el uso que hacen sus públicos a las redes sociales, que es del 100\% aunque su uso no es el adecuado (Simón Onieva, 2016).

- 2007 Comunidad Valenciana. El estudio de investigación realizado por Francisco Beltrán, La gestión de la nueva comunicación interna. Análisis de la aplicación de las tecnologías de la información en procesos de comunicación interna en las universidades de la Comunidad Valenciana, plantea un modelo de gestión de la nueva comunicación interna que toma como eje central los portales corporativos. Estos portales serán en un futuro - concluye el investigador- grandes repositorios de los contenidos informativos, por ello confrontó el uso de este canal en las universidades de la Comunidad Valenciana, instituciones con alta cultura tecnológica lo que las convierte en un laboratorio privilegiado para este ámbito de investigación. Vaticina que el modelo futuro de comunicación interna se apoyará en la gestión del conocimiento, en la segmentación de públicos y en la personalización de los contenidos.

Otros estudios de universidades españolas, donde se exponen casos concretos sobre comunicación interna expuestos en los artículos científicos de: García Orta (2012), Herranz de la Casa, Antonio D. Tapia Frade, Arturo Vicente Lázaro (2009) y Palencia-Lefler Ors (2008)

A nivel internacional en el ámbito latinoamericano, destacamos los trabajos sobre del perfil del comunicador interno de Abendaño y Duque (2016) en Ecuador; Nina Hanco (2015) sobre Google apps y comunicación interna; Osorio (2014) en Colombia quien hace un estudio so- 
bre la comunicación interna universitaria en el entorno digital; Trelles, L. (2005) en Cuba sobre Gestión de la comunicación interna en la universidad, y Benavente (2001) en Perú sobre noticias universitarias.

\section{Objetivos y fines}

La finalidad de la investigación ha sido aportar un estudio descriptivo sobre comunicación interna en las universidades de la Comunidad de Madrid. De otro lado, pretendemos contribuir con una investigación referencial para el seguimiento y evolución de la gestión de la comunicación interna, diferenciando la realidad del sector público y del privado.

Son objetivos generales del estudio: conocer el estado de la gestión de la comunicación interna en las universidades madrileñas con el fin de detectar debilidades y fortalezas.

Respecto a los objetivos específicos, estos son conocer de forma panorámica el estado de la gestión de la comunicación interna, es decir: si existe un responsable y una unidad de comunicación interna, cómo es este responsable, qué funciones realiza, la metodología que emplea; en definitiva, conocer de forma general, cuáles son las tendencias en comunicación interna y las fortalezas y debilidades en las universidades madrileñas.

La hipótesis central de la investigación persigue validar si existe presencia del comunicador interno en las universidades madrileñas y si éste asume las funciones propias de la disciplina. La gestión de la comunicación interna debe estar liderada por profesionales titulados, formados para tal función, que trabajen en equipo (Zapata Palacios, 1993). Así mismo, el comunicador interno no debe improvisar sino trabajar con una metodología: estudios de medición y diagnóstico de clima interno, políticas, segmentación de públicos, canales, herramientas, y planes de comunicación interna (García Jiménez, 1999).

\section{Metodología}

La técnica de investigación empleada ha sido una investigación descriptiva cuantitativa entre las 14 universidades ubicadas en la Comunidad de Madrid, las mismas que constituyen el universo de nuestro estudio. Se contactó con los/las responsables de comunicación interna, quienes tuvieron un plazo de tres semanas para remitir la respuesta, desde el 18 de enero al 1 de febrero de 2016.

El trabajo de campo se ha realizado a través de internet, vía correo electrónico, y la información correspondiente se ha recogido mediante el cuestionario cerrado con 21 preguntas, siendo la respuesta anónima.

En cuanto a la utilidad de la investigación, el tamaño de la muestra y el número de respuestas obtenidas garantizan suficientemente la representatividad de los resultados respecto a la realidad estudiada. Se ha obtenido una cuota de representatividad óptima (80\%) y, por lo tanto de alta utilidad en relación con los objetivos planteados.

En cuanto a los límites de estos resultados, el estudio hace referencia a las universidades de la Comunidad de Madrid.

Respecto a las fases de la investigación, se realizó en tres fases: documentación, ejecución y, resultados. La primera fase de documentación y estudio incluye la realización de un estudio documental con la finalidad de recoger antecedentes y de obtener referencias bibliográficas que ayuden a situar el estado de la cuestión y establezcan un marco de referencia que sirvan como primer paso a la investigación. En esta fase, se revisaron diversos artículos y estudios de investigación relacionados con el tema. Así mismo, en esta etapa se diseñó el cuestionario del que se 
hicieron las pruebas previas correspondientes del cuestionario. Por último, en esta fase se recopiló información de los responsables de comunicación interna, localizando nombre, teléfono y correo electrónico. Esta primera indagación nos permitió concluir que en la universidad madrileña la comunicación interna es liderada por mujeres. En el sector privado privadas localizamos 6 mujeres y 2 hombres y en el público, comprobamos que hay paridad, 3 hombres y 3 mujeres.

En la fase de ejecución se puso en marcha el cuestionario encuesta, el mismo que fue contestado vía correo electrónico y se hizo un seguimiento de sus respuestas por parte de los gestores de las universidades analizadas. En la última fase de resultados, analizamos todas las respuestas, obteniendo conclusiones generales y particulares distinguiendo amenazas, debilidades, fortalezas y oportunidades tanto en universidades privadas como en las públicas.

\section{Resultados y conclusiones}

\subsection{Análisis de resultados}

A continuación se exponen los resultados relevantes obtenidos que dan respuesta a los objetivos de investigación presentados en la introducción.

Población que participa. Se ha invitado a participar a todas las universidades de Madrid (14): públicas (6) y privadas (8). Al final, han accedido a participar 12 universidades (86\%), 6 públicas $(100 \%)$ y 6 privadas $(75 \%)$. Podemos considerar el estudio representativo de la situación de la comunicación interna en las universidades madrileñas.

Cuando una universidad decide que debe formalizar la $\mathrm{CI}$, se cuestiona otra pregunta: ¿quién debe ser el elegido? Si existe, es un dato revelador de la importancia que se concede a esta gestión. En las universidades madrileñas sí existe una figura institucionalizada del respon- sable de comunicación interna, tanto en las públicas como en las universidades privadas.

\section{Perfil de los responsables: antigüedad en el puesto}

El 75\% de los profesionales de comunicación interna de las universidades madrileñas (en adelante, UM) lleva 3 años o más trabajando en comunicación interna. En las públicas hay más expertos (dos tercios tienen más de 5 años de experiencia) y más nóveles (un tercio tiene menos de tres años), mientras que en las privadas, el 83\% tienen 3 años más de experiencia.

La tendencia es que los responsables de comunicación interna tienen una antigüedad superior a 5 años en el puesto. La antigüedad aporta experiencia y conocimiento. Cuanto antes se invierta en comunicación interna, la institución disfrutará de las ventajas de esta gestión y, por otro lado, el área o departamento, aprenderá de las experiencias y proyectos iniciados en comunicación interna. Según Puchol, los profesionales que tienen una antigüedad de 2 a 10 años han superado la etapa de incorporación al puesto de trabajo y se encuentran en una etapa profesional de crecimiento profesional (Puchol, 1997).

\section{Perfil de los responsables: edad}

Este dato está relacionado con la edad de los responsables de CI. La mitad de ellos tienen 35 o menos años. En las públicas dos tercios de los responsables tienen más de 35 años, mientras que en las privadas dos tercios de los responsables tienen entre 25 y 35 años

Es decir, los responsables de CI son mucho más jóvenes en las universidades privadas que en las públicas. La edad es un aspecto clave para el desempeño del puesto de comunicador interno, si tenemos en cuenta que tendrá que trabajar con diferentes perfiles. Las características perso- 
nales, hábitos, carácter y personalidad son un aporte al puesto.

También, tendríamos que tener en cuenta que las habilidades, conocimiento y experiencia mejoran con la edad. Otro aspecto importante, son los aportes propios de cada generación; en las empresas conviven y trabajan generaciones X, Y y Z. La tendencia es que este puesto lo ocupen personas entre 25 a 35 años, aunque también mayores de 55 años El conocimiento se ha convertido, para muchas organizaciones en uno de los activos más valiosos ya que la edad es sinónimo de saber hacer, ser y estar (Puchol, 1997).

\section{Perfil de los responsables: formación}

En cuanto a la formación que tienen los responsables de CI, la mitad (50\%) son periodistas. Un tercio (33\%) titulados en publicidad y relaciones públicas. Tan sólo un 8\% no tienen titulación universitaria. En las primeras la mitad son periodistas y el resto, publicidad (17\%), otros provienen de la rama de derecho (17\%) y alguno carece de titulación (17\%). En las privadas, la mitad son periodistas (50\%) y la otra son titulados en publicidad y relaciones públicas (50\%).

Las instituciones universitarias están llamadas a informar y comunicar, a ser más transparentes y más dialogantes. Por tanto, periodista y comunicador, son imprescindibles. El comunicador aplica estrategias periodísticas y domina la comunicación en todas sus variantes: «Es imprescindible, un profesional de la comunicación, que teniendo un gran poder de sintesis, una amplia fluidez mental, sea un experto y hábil comunicador» (Martín, 2006).

\section{Perfil de los responsables: vinculación laboral}

La vinculación laboral del responsable de CI es variada, como la propia realidad laboral univer- sitaria. Un tercio (33\%) es funcionario contratado, un cuarto (25\%) personal fijo y tan solo un $17 \%$ es eventual, como parece razonable al asumir la responsabilidad de la CI. Lógicamente, las categorías profesionales son diferentes en universidades públicas y privadas. Las públicas tienen un $50 \%$ de funcionarios contratados y un tercio (33\%) de funcionarios fijos. En las privadas, la mitad tienen otras categorías diferentes a las públicas y la otra mitad se la reparten en vinculaciones similares a las públicas.

Esta pregunta nos da pistas del conocimiento de la institución. La principal arma de un CI es el conocimiento de la cultura institucional: líderes, normas y procedimientos; en este caso, la estabilidad laboral es un factor a favor ya que, un personal fijo establece una relación más sólida con la institución, se siente más integrado, seguro y comprometido, lo que no podría ocurrir con un eventual. Los economistas Lindbeck y Snower elaboraron la teoría del empleo insiders/outsiders que explica la protección y desprotección del trabajador. Manuel Escudero en «Pleno empleo» explica que los trabajadores «outsiders» o temporales están abocados a la inestabilidad como horizonte de vida y que trabajan en la incertidumbre, ya que no se benefician de una igualdad de oportunidades y que serían despedidos cuando la economía fuese mal; los «insiders», sí disponen de medios de presión sobre la dirección empresarial, su poder de negociación es elevado y podrían pedir aumentos (Escudero, 1998).

\section{El perfil de los responsables: grado de dedicación}

La especial actividad de la universidad, hace que muchas veces los cargos de gestión se compaginen con la docencia. Esto ocurre a los responsables de CI de las UM: un tercio de ellos (33\%) 
mantienen actividad docente, mientras que el resto (66\%) tiene dedicación exclusiva.

Este dato indica que las autoridades universitarias, consideran que la CI no necesita de una jornada de 8 horas, sino que puede realizarse a media jornada. Un grave error.

La jungla del mercado laboral, competitiva y exigente les obliga a convertirse en profesionales multitarea, multi-tasking, teniendo que asumir varias tareas a la vez.

\section{Departamento o la unidad de comunicación interna}

El área de CI en las UM, en un 66\%, forma parte del área de la dirección de comunicación (en adelante, Dircom). Otras (17\%) dependen de Recursos humanos (en adelante, RRHH) o son autónomas (17\%). No obstante, en las públicas, las unidades autónomas suben hasta un tercio (33\%), en detrimento de las que dependen de Dircom (50\%). En las privadas no hay autonomía. Casi todas (83\%) dependen de Dircom, y el resto de RRHH (17\%).

Estos datos revelan la cultura institucional y el valor otorgado a la CI. Sobre este punto, quienes defienden que dependa de Dircom, lo justifican porque se trabaja en coordinación, por lo tanto, necesita situarse a nivel estratégico (Costa, 2012). Aunque, otros estiman que el área de CI, debe ubicarse en un departamento independiente para darle autonomía y que se gestione de forma independiente (Observatorio de comunicación interna, 2010).

\section{Nombre del área de comunicación interna}

En cuanto al nombre de la CI en las UM, como ocurre típicamente con las unidades de comunicación, la variedad es la que manda. El 42\% de ellas no tiene nombre y la mitad (50\%) tiene un nombre distinto $\mathrm{a}$ «departamento» $\mathrm{o}$ «unidad» de comunicación interna. En las privadas ninguna se llama así. Otros nombres utilizados, además de los dos citados propuestos, son: Área de comunicación interna; Delegada del rector para la comunicación institucional; Departamento de comunicación y relaciones institucionales; Dirección de comunicación institucional; Responsable de comunicación interna. La denominación, nombre que les distingue de otras áreas, les otorga cierta personalidad dentro de la estructura, le concede validez institucional, autoridad y relevancia.

\section{Dependencia orgánica del área de comuni- cación interna}

En cuanto a la dependencia orgánica, un tercio de los responsables de la CI en las UM dependen del Rector (o Presidente, en las privadas) y otro tercio depende del director de comunicación. El otro tercio tiene diversas dependencias. Esto cambia en las públicas, en las que la mitad (50\%) depende directamente del rector y el resto, variado. En las privadas, la mitad (50\%) depende del director de comunicación y el resto, variado.

¿Dónde debe ubicarse el área de comunicación interna? Debe ubicarse en el escalafón más alto, adjunto a los ejecutivos de mayor autoridad, son muchos los expertos que abogan por su más alta posición: Martín Fernando (2006), Piñuel (1997), García Jiménez (1999) o Costa (2001).

\section{Personal de apoyo}

La mitad (50\%) de los departamentos de CI en las UM están formados por entre 2 y 4 personas. Un 25\% de ellos son unipersonales y un 25\% tienen 5 o más personas en ellos. La situación varía en las públicas que se reparten por igual (33\%) las tres categorías establecidas. En las privadas, dos tercios de ellas (66\%) tienen entre 2 y 4 personas en el departamento. El resto se lo reparten por igual las otras dos categorías. 
Respecto al trabajo en equipo es relevante para el comunicador interno, puesto que no es lo mismo trabajar en soledad, que con las sinergias de un equipo. El trabajo en equipo permitirá obtener mejores resultados en tiempos cortos y, con una mejor calidad.

En cualquier caso, en comunicación interna la tendencia es organizar un Comité de comunicación interna, así como crear redes de corresponsales internos. Los equipos de trabajo multidisciplinares están alcanzando resultados significativos. En el caso de la universidad se necesita de un equipo potente para poder abordar los proyectos necesarios.

\section{Presupuesto de comunicación interna}

Respecto al presupuesto con el que cuentan, el 58\% de las UM dice no contar con presupuesto, mientras que el 25\% dice que es menos de 30.000 euros anuales y un 17\% dice que es mayor de 50.000€. En las UM públicas el porcentaje de las que no tienen presupuesto aumenta hasta dos tercios de ellas (66\%) y baja en las UM privadas hasta el 50\%. El presupuesto asignado a la comunicación interna revela la apuesta decidida por esta disciplina.

No basta tener buenas intenciones, hay que invertir y dedicar partidas presupuestarias, de lo contrario habrá limitaciones de acción para el área de CI. La rentabilidad está asegurada ya que la institución obtendrá beneficios en intangibles: la marca interna, en el sentimiento de pertenencia, en una plantilla comprometida. La tendencia es apenas tener asignadas partidas para la comunicación interna.

\section{Gráfico 1 • Presupuesto del área de comunicación interna}

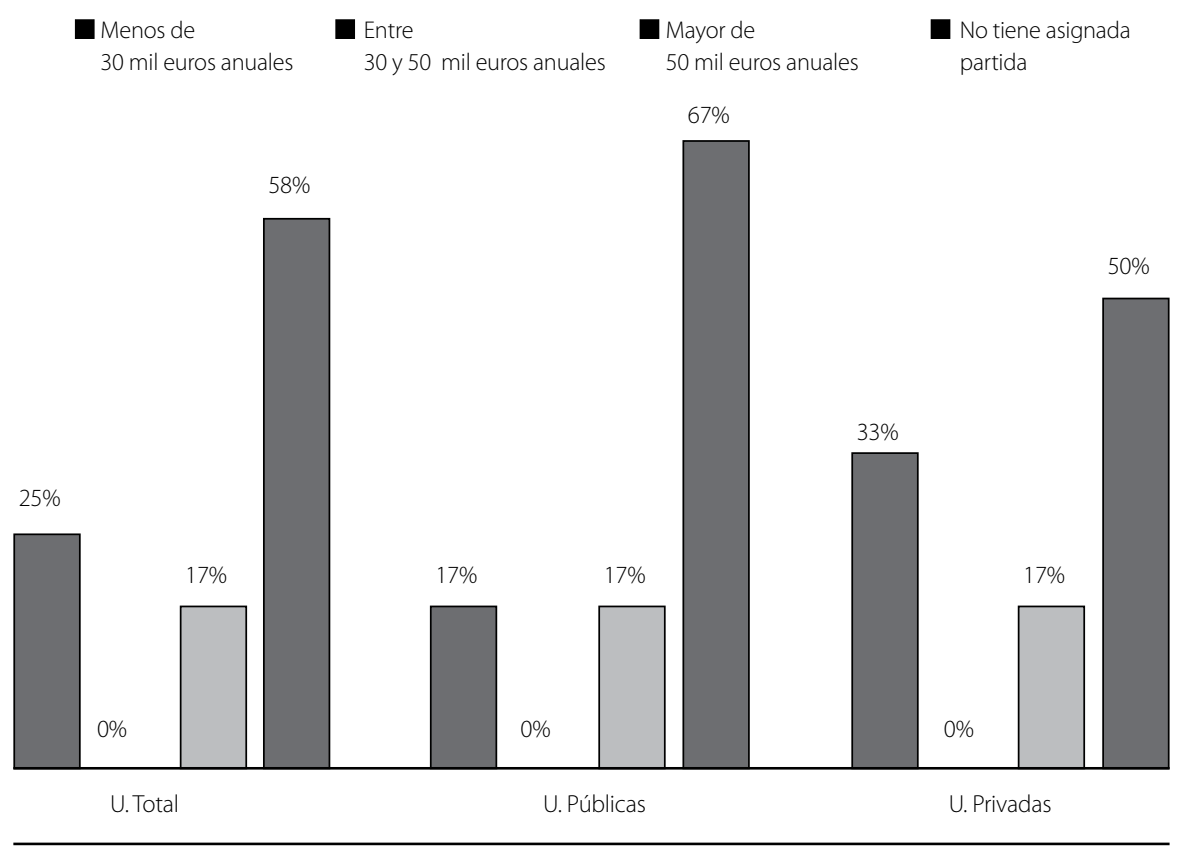




\section{Políticas y gestión de la comunicación interna}

La mayoría (66\%) de las UM tienen política de CI y está alineada con las de comunicación externa (en adelante, $\mathrm{CE}$ ). El resto o no tienen política de CI (17\%), o la tienen pero no está alineada con las políticas de CE (17\%). En las UM públicas alinean más sus políticas, en un 66\% de ellas tienen política de CI y está alineada con la CE.

Las políticas deben estar recogidas y delimitadas en un documento oficial que debe divulgarse entre todo el personal e ir en sintonía con la comunicación externa. El beneficio las políticas reside en que las organizaciones necesitan de unos principios generales para canalizar sus comunicaciones internas, y conseguir mensajes más eficientes y coherentes.

\section{El plan de comunicación interna}

La mayoría de las UM (83\%) trabaja la CI desde un plan de CI. Tan sólo una minoría (17\%) no tiene plan. En este apartado coinciden por igual públicas y privadas. Aunque las universidades madrileñas trabajan con planes de comunicación, desconocemos si son estratégicos.

Los planes de comunicación interna deben ser estratégicos y estar recogidos en un documento que incluya políticas, programas, acciones y estrategias. Permiten alinear el discurso al objetivo de la institución, crear mensajes claves para los diferentes colectivos y facilitar y difundir un discurso coherente.

\section{Objetivo prioritario de la comunicación interna}

El objetivo de la CI para las UM es variado. Para el $42 \%$ de ellas se trata de transmitir y dar a conocer el proyecto institucional. El 25\% afirma que su objetivo es informar sobre la marcha institucional y atender las expectativas informativas del personal. En las UM estos dos objetivos tie- nen el mismo peso (33\%). En las UM privadas el objetivo de transmitir y dar a conocer el proyecto institucional es el más común (50\%) y los demás objetivos se reparten por igual en porcentaje minoritario (17\%).

Conocer el objetivo prioritario nos permite conocer para qué sirve la comunicación interna. Puede servir para comunicar el proyecto institucional, para gestionar intangibles: marca interna, talento, reforzar los valores y hacerlos vivir. También para generar debate sobre la calidad educativa y el posicionamiento de la universidad, objetivo imprescindible.

\section{Rol de la comunicación interna}

Cuando se les pregunta sobre el rol de la CI en las UM, la respuesta es variada. Para un tercio de ellas (33\%) es la gestión del cambio, mientras que un $25 \%$ piensa que debe fomentar la transparencia. Los roles son muy dispares en las UM públicas, sin una clara coincidencia entre ellas. Sin embargo, en las UM privadas parece que lo tienen mucho más claro: la mitad (50\%) piensan que es la gestión del cambio y un tercio (33\%) que debe fomentar la transparencia. Una minoría (17\%) cree que ha de gestionar el orgullo de pertenencia.

Un rol importante del comunicador interno es promover la transformación institucional para hacer realidad la transparencia y así gestionar la confianza. Otro rol clave es ser un facilitador y un promotor de la innovación en la universidad.

\section{Estudios de segmentación de públicos}

Tres cuartas partes de las UM (75\%) trabajan con estudios de públicos internos segmentados. Este porcentaje aumenta en las públicas hasta el 83\% y disminuye en las privadas hasta el $66 \%$.

La segmentación de públicos se refiere a los elementos comunes que tienen los diferentes 
públicos potenciales a quienes destina mensajes el área de comunicación interna. Entre otras ventajas, permite conocer de modo general, el perfil de personas que integran la institución, analizar cada uno de los grupos y poder centrar una estrategia de mensajes, más eficaz; así mismo realizar acciones específicas que satisfagan el target concreto.

Las empresas e instituciones deben conocer y reconocer la diversidad de público y según sus características enviarles mensajes claves. Cada público tiene unas expectativas particulares y en función de esas expectativas se formará una imagen interna de la organización. Un estudio de segmentación profesional es esencial para focalizar los esfuerzos de CI.

\section{Diagnóstico de clima interno}

La mayoría de las UM los realizan, aunque el porcentaje varía cuando lo diferenciamos por universidades públicas y privadas. Hasta un tercio (33\%) de las públicas no los realiza, mientras que el $100 \%$ de las privadas los hace. En estas últimas, una mitad de ellas los hace regularmente y la otra, esporádicamente.

Todo empieza o debe empezar en comunicación interna con un diagnóstico. Es fundamental escuchar para conocer las percepciones de los colaboradores respecto a políticas institucionales, al liderazgo, al compañerismo, etc., aspectos decisivos para el aprovechamiento del talento.

\section{Evaluación de la comunicación interna}

La herramienta más utilizada para medir la eficacia de las acciones de CI son las encuestas que utilizan el $42 \%$ de las UM, mientras que las auditorías son minoritarias (17\%). Hasta un tercio de las universidades (33\%) no realiza ninguna actividad para medir la eficacia de la CI. Esto es muy diferente según la titularidad: el 50\% de las UM no realiza ninguna actividad para medir la CI. En las públicas es sólo un 17\%

Evaluar es la última fase del proceso que abarca todos los programas y actividades implantadas en la empresa y que se han puesto en marcha desde el plan anual de comunicación interna. Sin embargo, en la práctica al parecer nunca hay tiempo para ello.

\section{Canales más utilizados}

En cuanto a los canales más utilizados para la CI, las UM usan un abanico grande de ellos, siendo los más utilizados las herramientas digitales (25\%) y los canales propios de TV, radio, prensa... (25\%). El canal más utilizado en las UM públicas son las herramientas de escucha (33\%), repartiéndose por igual el resto de las otras. Aquí también hay una gran diferencia con las UM privadas que utilizan mayoritariamente los canales propios de TV, radio, prensa... (50\%). También utilizan más las herramientas digitales (33\%) y minoritariamente (17\%) el correo electrónico, medios utilizados para crear comunidad y facilitar la colaboración.

Los canales sean orales, escritos, audiovisuales o electrónicos, son necesarios como vehículos transmisores de mensajes. Son imperiosos para compartir conocimientos y difundir la cultura corporativa, los planes y actividades institucionales. El contenido de los mensajes condiciona el canal utilizado, conseguir impacto en los mensajes es el gran reto.

\section{Acciones de comunicación interna más utilizadas}

Las acciones de CI más utilizadas por las UM son los programas de reconocimiento (33\%). En el resto ya no hay mucha coincidencia. Algunas destacan los programas de responsabilidad so- 
cial interna (17\%) o los planes de formación y desarrollo (17\%).

Todas estas acciones son importantes, sin embargo en el caso de la universidad no deberían descuidarse las acciones de responsabilidad social interna, ni la formación.

\section{Tipo de comunicación más importantes}

Para el 50\% de ellos, lo más importante es la comunicación directa al personal a través de los canales institucionalizados. Para un 25\% la comunicación interpersonal y grupal entre jefes y colaboradores. En las UM públicas en un $66 \%$ promueven la comunicación directa al personal, mientras que en las UM privadas el $50 \%$ apuesta por la comunicación interpersonal y grupal.

Los tipos de comunicación se refieren a la forma como conectan con sus públicos internos, la comunicación interpersonal es una de las más importantes ya que se acoge con agrado que un jefe, reserve tiempo para hablar directamente, de forma privada con cada colaborador.

\section{Conclusiones}

Todas las universidades madrileñas asumen la responsabilidad de comunicar internamente. Por tanto, quedó demostrada la hipótesis de que existe un responsable de comunicación interna en las universidades madrileñas; en la mayoría de casos se trata de un titulado universitario graduado en Periodismo, Publicidad o Relaciones Públicas. Estos profesionales trabajan en equipo y desempeñan sus funciones desde una posición holística y estratégica, son en su mayoría directivos. Trabajan con una metodología propia de la disciplina como es el diseño de mapa de públicos y con planes de comunicación interna. Asumen como objetivo principal transmitir y dar a conocer el proyecto institucional y han incorpo- rado a sus recursos las nuevas tecnologías y las redes sociales

Desde estas características se puede afirmar que la comunicación interna institucional universitaria, tanto en el sector público como privado, avanza y se consolida en las universidades madrileñas respecto a 2005. Sin embargo, presentan ciertas debilidades que deben corregirse.

Hemos detectado 10 debilidades en la gestión de CI de las universidades madrileñas y son: 1) Sus responsables son profesionales multitarea; 2) En la mayoría de casos, desempeñan sus responsabilidades sin contrato fijo, desde un contrato eventual; 3) El personal de apoyo del que disponen fluctúa de dos a cinco personas, un número insuficiente para abarcar las múltiples tareas que demanda la gestión de la comunicación interna; 4) El área de comunicación interna no tiene nombre en algunas universidades, hecho que le podría restar identidad y reconocimiento; 5) No alinean sus políticas de comunicación interna con la política de comunicación externa de la universidad; 6) Están centradas en la gestión del cambio y en la transformación institucional, olvidando el objetivo de mejorar la calidad docente y promover la innovación educativa; 7) Se descuida la comunicación interpersonal y grupal así como los eventos sociales internos; 8) No se realizan estudios de diagnóstico de clima laboral; 9) Tienen bastante abandonadas las auditorías de comunicación interna; 10) Su mayor limitación es la escasa asignación económica.

Respecto a estas carencias, omisiones y debilidades corresponde a cada universidad en particular, abordar nuevos proyectos para afrontar sus propias debilidades. En este sentido, sería recomendable aprender de las buenas prácticas o casos de éxito en comunicación interna institucional. 
El progreso necesario en comunicación interna institucional universitaria podría basarse en cuatro áreas de mejora: 1) Asignar mejores presupuestos; 2) Implicar a las altas autoridades; 3) Emprender proyectos para empoderar la marca universidad, y 5) Implantar diagnósticos y mediciones de toda acción y proyecto realizado.

1) Asignar mejores presupuestos implica incrementar el presupuesto en comunicación interna y establecer mediciones KPIs y ROI a fin de medir la rentabilidad de la inversión. Cada área de comunicación interna podría establecer unos indicadores que demuestren el dominio, control y optimización de todos sus proyectos en comunicación interna; es recomendable medir la eficacia de la inversión en comunicación interna a partir de Indicadores Clave de Desempeño (KPIs: Key Performance Indicators), aprovechando las herramientas digitales gratuitas. Así también, convendría hacer un seguimiento de la inversión eficiente en comunicación interna y calcular el ROI (Return On Investment) de su gestión, para demostrar que la inversión en comunicación interna aportó a la eficiencia de la universidad y consiguió beneficios. 2) Implicar a las altas autoridades en proyectos y campañas de CI es un factor clave para conseguir impacto y notoriedad en cualquier proyecto de comunicación interna; convendría también, movilizar al personal, creando redes de corresponsales de comunicación interna y organizando comités de comunicación interna en las diferentes facultades. 3) Recomendamos empoderar la marca universidad con la finalidad de generar compromiso, visibilidad y de modo que contribuyan a la mejora de reputación; en este sentido se podría aprender de las buenas prácticas implantadas por otras universidades. 4) Por último, consideramos imprescindible realizar mediciones y evaluaciones periódicas para saber lo que está funcionando y lo que no. De esta manera, se podrían realizar con una periodicidad bianual, estudios de clima laboral y auditorías de comunicación interna, con miras a evaluar la eficacia de los proyectos de comunicación interna, establecer correcciones, reconducir desviaciones y optimizar canales y procesos.

Salvo las carencias y mejoras señalas, concluimos que el estado de la comunicación interna en las universidades madrileñas aunque tiene más debilidades que fortalezas, se encuentra en una fase de despegue, con grandes perspectivas de futuro. Todo depende del protagonismo que las altas autoridades universitarias le quieran otorgar. Lo importante es que los primeros e importantes pasos de establecer un área y asignar a un responsable ya se han dado. 


\section{ANEXO 1}

Cuestionario aplicado a universidades públicas y privadas de la Comunidad de Mdrid

1. ¿Trabaja en una universidad?

$\square$ Pública

2. ¿Cuántos años lleva trabajando en el área de comunicación interna?

Menos de 3 años

Entre 3 y 5 años

Más de 5 años

3. El área de comunicación interna en la que usted trabaja:

Forma parte del Departamento de RRHH

$\square$ Forma parte de la Dirección de Comunicación

Es una unidad de comunicación interna autónoma

4. ¿Cómo se llama su área de comunicación interna?

Unidad de Comunicación interna

Departamento de comunicación interna

No tiene nombre

$\square$ Otro...

5. El área de comunicación interna en la que usted trabaja está ubicada respecto al organigrama...

Adjunto al máximo responsable (Rector)

Adjunto al Director de Comunicación

$\square$ Pertenece a RRHH

Es una área independiente y pertenece a otro departamento

$\square$ Otro...
6. ¿Qué tipo de vinculación tiene con la universidad?

$\square$ Personal fijo (funcionario público o de carrera)

$\square$ Personal contratado (funcionario contratado)

$\square$ Personal eventual ( funcionario interino)

$\square$ Otro...

7. ¿Cuál es su rango de edad?

Menor de 25 años

Entre 25 a 35 años

$\square$ Entre 36 a 45 años

$\square$ Entre 46 y 54 años

$\square$ Mayor de 55 años

8. ¿Cuál es su titulación universitaria?

$\square$ No tiene titulación universitaria

$\square$ Empresariales / Económicas

$\square$ Periodismo

$\square$ Publicidad y RRPP

$\square$ Sociología

$\square$ Derecho

$\square$ Otro...

9. Además de responsable de comunicación interna, ¿trabaja como docente en la universidad?

$\square$ Sí, además trabajo como docente

$\square$ No, tengo dedicación exclusiva a trabajos administrativos

10. ¿Cuántas personas integran el área de comunicación interna?

1 persona

Entre 2 y 4 personas

5 personas o más 
11. ¿Existe una política de comunicación interna? ¿Está alineada con la comunicación externa?

$\square$ Si, existe pero no está alineada con la comunicación externa

$\square$ Sí, está alineada con comunicación externa

$\square$ No existe política de comunicación interna

12. ¿Realiza su institución estudios de diagnóstico de clima interno?

$\square$ Sí, de forma periódica (anual, semestral...)

$\square$ Sí, esporádicamente

$\square$ No, nunca los hemos realizado

13. ¿Trabajan en comunicación interna con estudios de segmentación de públicos internos?

$\square$ Sí

$\square$ No

14 ¿Trabajan la comunicación interna desde un plan de comunicación interna?

$\square$ Sí

$\square$ No

15. ¿Cuál es el rol más importante que cumple la comunicación interna en su institución? Los roles más habituales que se asumen desde el área de CI son:

Es una herramienta para

$\square$ La gestión del cambio y transformación institucional

$\square$ Gestionar el sentimiento de orgullo de pertenencia

$\square$ Fomentar la transparencia y confianza

$\square$ La mejora de la calidad docente e innovación

$\square$ Desarrollar una cultura participativa

$\square$ Otro...
16. En su institución, ¿cuál es el objetivo prioritario en comunicación interna?

$\square$ Transmitir y dar a conocer el proyecto institucional (misión, visión, valores)

$\square$ Informar sobre la marcha institucional y atender las expectativas informativas del personal que integra la universidad

Velar por los activos intangibles: marca y reputación interna

Mejorar la participación y coordinación entre los diferentes departamentos de la universidad

$\square$ Generar debate para mejorar la calidad educativa y posicionamiento de la universidad

$\square$ Otro...

17. ¿Qué canales de comunicación interna tienen más importancia en su universidad?

$\square$ Herramientas de escucha ( encuestas, auditorías de comunicación interna)

$\square$ Canales tradicionales ( reuniones, tablones de anuncios, buzón de sugerencias, revistas impresas)

$\square$ Canales propios de TV, radio, prensa

$\square$ Herramientas digitales ( intranet, foros, blogs, wikis, )

Correo electrónico

$\square$ Canales digitales (Sistemas de mensajería electrónica SMS, Whatsapp y/o redes sociales corporativas)

$\square$ Otro...

18. En su actividad o plan de comunicación interna, ¿qué acciones son las más importantes?

Planes de acogida y programas de despedida del personal 
Programas de responsabilidad social interna y voluntariado

$\square$ Programas de formación y desarrollo personal

Programas de reconocimiento

$\square$ Eventos sociales internos (aniversario, Navidad...)

$\square$ Otro...

19. En su universidad, ¿a qué tipo de comunicación se le concede mayor importancia?

Comunicación directa al personal a través de los canales institucionalizados (entrevistas, correo electrónico, revistas, etc.)

$\square$ Comunicación interpersonal y grupal (entre jefes y equipo de colaboradores)

$\square$ Comunicación interdepartamental (Comités y comisiones de trabajo)

$\square$ Comunicación con los líderes de la institución (reuniones con altos representantes, eventos internos)

$\square$ Otro...
20. ¿Realizan alguna actividad para evaluar y medir la eficacia de la comunicación interna?

$\square$ Sí, encuestas.

$\square$ Sí, auditorías

$\square$ Sí, encuestas y auditorías

$\square$ No.

$\square$ Otro...

21 ¿Qué presupuesto tiene asignado para comunicación interna?

$\square$ Menos de 30 mil euros anuales

$\square$ Entre 30 y 50 mil euros anuales

$\square$ No tiene asignada partida 


\section{Bibliografía}

Abendaño Ramírez, M. y Duque Rengel, K. (2016). El perfil del DirCom Interno en el Ecuador, Universidad de Loja. Recuperado el 23 de mayo de 2017, de: Asociación de Directivos de Comunicación (2013). Manual de Comunicación. Madrid: Dircom 2013.

Asociación de Directivos de Comunicación. DIRCOM. (2010). Estado de la comunicación en España (2010). Recuperado el 15 de mayo de 2017 de: https://mouriz.files.wordpress.com/2010/05/estado-comunicacic3b3n.pdf

Beltrán Fernández, F. (2007). La gestión de la nueva comunicación interna. Análisis de la aplicación de las tecnologías de la información en los procesos de comunicación interna de las universidades de la Comunidad Valenciana. Universidad Jaime I, Tesis Doctoral. (Enlace http://www.tesisenred.net/handle/10803/10464).

Cabanas, C. y Soriano, A. (2014). Comunicar para transformar. Madrid: Editorial LID.

Cardozo Vale, S.; Vásquez, M. Herramientas de comunicación interna en la Universidad de Los Andes, Trujillo. Visión Gerencial, [S.1.], n. 1, p. 63-80, ene. 2015. Recuperado el 23 de mayo de 2017, de: <http://erevistas.saber.ula.ve/index.php/ visiongerencial/article/view/5390/5183>.

Carrascosa, J. (2003). Comunicacción: de la sociedad de la información a la sociedad de la comunicación. Madrid: Arcadia S.A.

Costa, J. (2001). La comunicación en acción: informe sobre la nueva cultura de la gestión. Barcelona: Editorial Paidós.

Costa, J. (2014). El DirCom hoy: dirección y gestión de la comunicación en la nueva economía. Barcelona: Joan Costa Institute.

Crovi. (2005). La articulación medios-universidad en la distribución social del conocimiento (UNAM, México).

Estudio citado en:

https://www.ull.es/publicaciones/latina/2008/22_36_ UPF/Manel_Palencia.html

Escudero, M. (1998). Pleno empleo. Madrid: Ed. Espasa Calpe. Espinosa Osorio, F. (2014). Comunicación interna en el entorno digital de la Universidad de Ibagué, Revista Comunicación, No. 31 p. 55 - 66 Medellín-Colombia. Enero-diciembre de 2014, ISSN 0120-1166 / ISSN (en línea): 2390-0075

García Jiménez, J. (1998). La comunicación interna. Madrid: Ediciones Díaz de Santos.
García Orta, J. (2012). Comunicación Interna y Universidad: una aproximación teórica, Trabajo de fin de máster facultad de Comunicación de la Universidad de Sevilla. Enlace consultado 23 de mayo de 2017: http://fama2.us.es/fco/tmaster/tmaster34.pdf

Gómez Quijano, A. (2010). Comunicación para ejecutivos. Las tres distancias de la comunicación en las organizaciones. Madrid, EUNSA, segunda edición.

Gondrand, F. (1978). La Práctica de la Información en la Empresa. Madrid: RIALP.

Herranz et al.(2009). La comunicación interna en la universidad. Investigar para conocer a nuestros públicos. Revista Latina de Comunicación Social, 64, páginas 262 a 274. La Laguna (Tenerife): Universidad de La Laguna, recuperado el 29 de mayo de 2017, de: http://www.revistalatinacs.org/09/ art/23_822_30_Valladolid/Herranz_et_al.html DOI: 10.4185/RLCS-64-2009-822-262-274

Losada Díaz, J. C. (2002). Prensa e imagen corporativa en la universidad: los públicos internos. Murcia: Universidad Católica San Antonio de Murcia.

Martín, F. (2006). Comunicación Institucional y empresarial. Madrid: Universitas.

Nina Hanco, H. (2015). Software como Servicio Google Apps para la Comunicación Interna en la Universidad San Antonio Abad del Cusco. Tesis para optar la Maestría no publicada. Universidad San Antonio de Abad del Cuzco (Perú), Facultad de Ingeniería.

Observatorio de comunicación interna

(2012). Redes Sociales, Social Media y Entornos Digitales en Comunicación Interna.

(2009). Estudio sobre la Comunicación interna en Empresas Privadas y las Administraciones Públicas Españolas.

(2005). I Estudio de Comunicación Interna en la Administración Pública. Madrid.

(2002). III Estudio de Comunicación Interna Liderazgo en Comunicación Interna. Madrid. Disponible en: http://atrevia. com/observatorio-de-la-comunicacion-interna/ Consultado el 29 noviembre de 2016.

Ongallo, C. (2007). Manual de comunicación: guía para gestionar el conocimiento, la información y las relaciones humanas en empresas y organizaciones, Madrid: Dykinson, S.L. 
Palencia-Lefler Ors, M. 2008. La incomunicación interna en la universidad española. Universidad Pompeu Fabra. Recuperado el 29 de mayo de 2017, de: https://www.ull.es/publicaciones/latina/_2008/22_36_UPF/Manel_Palencia.html

Piñuel Raigada, J.L. (1997). Teoría de la comunicación y gestión de las organizaciones, Madrid: Síntesis.

Puchol, L. (1997). Dirección y gestión de recursos humanos. Madrid: Díaz de Santos.

Romero Sanz, S. y Calero de la Paz, R. (2005). Análisis del Marketing interno en las universidades de la Comunidad de Madrid, en Revista DIALNET UNIRIOJA.ES. Recuperado el 5 de abril de 2017.

Saló, N. (2005). Aprender a comunicarse en las organizaciones. Barcelona: Editorial Paidós.

Simón Onieva, J. E., Gabinetes de comunicación universitarios y redes sociales. Estudio del uso de las redes sociales por las uni- versidades públicas andaluzas. Tesis doctoral, fecha de lectura 8 de enero de 2016, Universidad de Almería. Enlace consultado el 23 de mayo: https://www.educacion.gob.es/teseo/cr eatepdf?origen $=3$ \&idFich $=400780$

Trelles L. (2005). Gestión de comunicación universitaria: razones, ámbitos y modos. Universidad de La Habana, Cuba, citado en: García Orta, Juan (2012). Comunicación Interna y Universidad: una aproximación teórica.

Zapata Palacios, L. (2016). Industria de la Comunicación y Economía Digital: guía básica del DIRCOM, Barcelona: UOC. Zapata Palacios, L. (1993). La comunicación interna fundamentos para su organización y eficacia. Tesis doctoral no publicada. Universidad Complutense de Madrid, Facultad de Ciencias de la Información. Madrid. 